\title{
RASPBERRY PI-BASED ACCESS CONTROL USING FACE RECOGNITION
}

\author{
Ondrej KAINZ*, Ján DROZD**, Miroslav MICHALKO ${ }^{* * *}$, František JAKAB ${ }^{* * * *}$ \\ * Department of Computers and Informatics, Faculty of Electrical Engineering and Informatics, Technical University of Košice, \\ Slovak Republic, tel. +42155602 7017, E-mail: ondrej.kainz@tuke.sk \\ ** Department of Computers and Informatics, Faculty of Electrical Engineering and Informatics, Technical University of Košice, \\ Slovak Republic, E-mail: jan.drozd@student.tuke.sk \\ *** Department of Computers and Informatics, Faculty of Electrical Engineering and Informatics, Technical University of Košice, \\ Slovak Republic, tel. +421 55602 7080, E-mail: miroslav.michalko@tuke.sk \\ ${ }^{* * * *}$ Department of Computers and Informatics, Faculty of Electrical Engineering and Informatics, Technical University of Košice, \\ Slovak Republic, tel. +421 55602 2039, E-mail: frantisek.jakab@tuke.sk
}

\begin{abstract}
The aim of the research is the proposal and experimental implementation of a solution based on Raspberry Pi that is to allow face recognition with subsequent user notification. Proposal of whole system discusses the possible negative factors that can endanger functionality of the system. Once the face is recognized or not recognized, the message is sent to the administrator and access is permitted or denied. This represents the elevated level of system hardening. The system in this research is built on histogram of oriented gradients (HOG) and neural network (NN). Testing of the software system solution has been also carried out and proves the usability with detection and recognitions rates above $90 \%$. The implemented system may be used as a part of complex IoT (Internet of Things) security solution.
\end{abstract}

Keywords: Computer vision, face detection, face recognition, Internet of Things, Raspberry Pi.

\section{INTRODUCTION}

The things that surround us are becoming more interconnected due to technologies that became the main aspect of the most industries. Digital devices help to collect, analyze and monitor data from surroundings and are able to communicate between each other through the Internet, thus may be referred as Internet of Things (IoT) devices. Public sector, such as hospitals, utilizes sensors to effectively monitor the patients or governments and cities can monitor the traffic volume or the air pollution rate. More on IoT and its models and possible solutions is described in [1].

Face recognition is getting large attention as well. It can be used for accelerated human identification. Just imagine systems allowing recognition of a client right after arrival to a bank or insurance company. Another example give advertising companies that develop advertising boards, which adjust the content of the passing by person. Thus, such board adjust to age, gender and even personal style after the face and body analysis.

Commercial smart access control systems, e.g. doorbells, are created in analogous way the. They provide the information to an owner of a property. These smart doorbells provide the ability to communicate with a visitor without direct confrontation. The middle element of communication is the mobile device and the doorbell.

\section{METHODS FOR FACE DETECTION AND RECOGNITION}

The basic step when detecting any object from the image is to determine its features. For the computer each object is set of features, such as edge, arc, brightness etc. Detecting features consists of obtaining the appropriate characters or points of an object that is detected. Further are analyzed the most popular techniques for face detection and recognition.

\subsection{Histogram of Oriented Gradients}

In this technique, the image is subdivided into smaller parts that make it easier to extract useful information and discarding unnecessary. This part of image is called the descriptor. In the Histogram of Oriented Gradients (HOG) feature descriptor, the histograms of directions of gradients are used as features. Gradients of an image are useful because the magnitude of gradients is large around edges and corners.

Recent research by Hafiz et al. [2] utilized HOG along with CNN (Convolutional Neural Networks) and proved $89 \%$ accuracy of recognition. Also, comparative research [3] utilizes HOG with CNN and also with ANN (Artificial $\mathrm{NN}$ ) and proves this technique to be useful complement in the recognition process.

\subsection{Haar Classifiers}

Object detection using Haar classifiers is an effective method proposed by Paul Viola and Michael Jones in their research published in [4]. Haar's classifiers focus on the square properties of the object. Object properties use the change in contrast values of adjacent pixels instead of using pixel intensity. Changes in contrast between pixel groups are used to determine light and dark areas. Haar's features can be easily scaled by zooming in and out of the pixel groups. Thanks to this, features can recognize objects of many sizes. Haar classifiers use 3 types of features:

- 2- rectangle features,

- 3- rectangle features,

- 4- rectangle features.

Haar classifiers along with addition weak classifiers were utilized in the research by Cuimei et al. [5] for detection of human face, authors yield the accuracy to be approx. 80 
percent in case of sole Haar classifiers, and 98 percent using their proposed solution.

\subsection{Support Vector Machine}

Support Vector Machine (SVM) is a popular machine learning tool that belongs to the category of kernel methods. Authors in [6] state that SVM "has originated from statistical learning theory for classification and regression problems". What SVM actually does is maximization of margin between two classes via hyperplane. This hyperplane is placed between them, i.e. best separation of these two classes is achieved by hyperplane.

Huang in [7] refers to SVM as to hyperplane classifier, further he notes that providing that SVM is not able to separate one class from other in original input space, SVM then maps this data to $\mathrm{N}$-dimensional feature space, where $\mathrm{N}$-dimensional hyperplane is created to separate these classes effectively. For case of data that cannot be linearly separated in its original space, i.e. no optimal hyperspace exists, the nonlinear SVM may be used.

One of the popular utilization of SVM is in the field of image processing, specifically also face recognition. One of such research was carried out by [8], authors however focused on facial expressions, i.e. anger, disgust, fear, happiness, sadness and surprise, thus six classes were to be considered - using Cohn-Kanade database with actual accuracy of 97.75 percent and second accuracy of 93.7 percent for Facial Action Units (FAUs) recognition, both using SVM. Rather recent research on face recognition is discussed in [9]. Again, authors utilized face database of totally 120 images of 15 persons and describe this method as a novel for SVM multi-classification, its essence is in error-coding codes principle that turns it into twoclassification problem. However, common method for multiclassification problem solution is to decompose it into series of two-classification problems.

Recent survey [10] yield SVM as still popular and efficient in terms of face recognition with the accuracy over 95 percent.

\subsection{Neural Networks}

Artificial neural networks or simply neural networks $(\mathrm{NN})$ are based on the real biological neural structures. Idea of $\mathrm{NN}$ is over 50 years old, however many new models and networks have been lately proposed. Authors in [11] refer to these networks as distribution free computational methods with non-linear nature, thus have the potential to solve complex issues of non-linear nature. Generally, network is consisted of neurons that form input layer, hidden layer or output layer. Many different network models and network approaches were developed over the years, most commonly used are presented by [11], e.g. single/multilayer feedforward, fully recurrent etc. Same authors generally distinguished two types of neural networks: Feed forward (single or multilayer neural network, single layer utilize hidden neurons where input units may or may not be connected to output units, however in multilayer are signals spread from input units to other output units in forward (one) direction, i.e. no cycle is formed). Recurrent or feedback (units here are connected one to another, cycles in topology are present, also training of these networks is rather more complex).

$\mathrm{NN}$ is utilized in various areas of research, in terms of face recognition, the survey [10] indicates its accuracy to over 98 percent.

\section{POSPOSAL OF FACE RECOGNITION SYSTEM}

Proposed solution contains multiple components including camera, its output will be used to detect and recognize face and check a database for historical data. Following the prior analysis, the Raspberry Pi Model 3 B+ was selected as the main system component for execution of all the processes. Proposed system considers the following steps:

1. Entry of trusted people to the system: Represents the known people to be recognized by the system.

2. Capture the face of visitor as a static image: The image is saved for next processing.

3. Face detection: Utilizes HOG algorithm. The goal is the detection of the face or multiple faces from the image. At this point, the information about the number of detected faces will be the output.

4. Extraction of facial points from the visitor's image: At this stage, the values of the points of all the faces will be stored.

5. Face recognition: In this step, these extracted face point values will be compared with each other using the Euclidean distance to determine into what degree they match. If the values match, the face is recognized.

6. Assigning name to a recognized face: After a successful recognition, a recognized person will be assigned a name. Unrecognized person will be given description "unknown person".

7. Notification of administrator.

8. Access control: Permission or denial of access from the remote location.

9. Electronic door lock opening (experimentally implemented in the form of temporal LED light).

The basic library used in the system for face detection and recognition will be faceRecognition (based on the dlib library). SD card represents the memory and also contains a database of known faces.

As previously mentioned, the HOG method is to be used for face detection based on hardware assumptions due to accuracy and speed of work. Successful face detection is necessary for the next recognition process. Another precondition for face recognition is the initial initialization of images from a database of known faces. For each known person, several photographs will be provided, while each photo for each person will be stored separately. When taking these images, possible negative factors that can endanger functionality of the system have to be considered. Thus, we should include images under various lightning conditions, with various distances from the camera and various face angles.

For the purposes of face recognition, each photo from database during initialization will go through trained neural network (which is pretrained by the dlib library on a dataset of three million images). The values obtained will be stored and compared to the visitor's face values in the final phase 
of the algorithm. Based on this process, the Euclidean distance will be used to compare, both faces and information about recognized or unrecognized face will be given as an output.

Based on the testing, training of NN on a Raspberry Pi is not recommended due to insufficient computing power. A suitable solution is to use a pre-trained network. Such a $\mathrm{NN}$ training process requires a large amount of computational power and input data. As soon as the $\mathrm{NN}$ is trained, it can generate measurements for any face.

The proposed recognition system generates the output accessible to the user, this output will represent the image with recognized or unrecognized face. Notification is sent to the mobile device of administrator along with a text output containing the name of the recognized person. The purpose of the whole communication is to provide double authentication.

As part of the system, an electronic lock, which is not the topic of this research, should be opened after the user has sent a message "Open" to unlock the door. In case of proposed system, instead of an electronic lock, the LED signaling is used.

\section{EXPERIMENTAL IMPLEMENTATION}

The main component of a system is prototyping board Raspberry Pi Model $3 \mathrm{~B}+$. Raspbian OS is being used for Raspberry Pi. System has been implemented in Python language in version 3.6.5.

\subsection{Initialization}

Face recognition process requires to initialize all the images in the database of known faces. Once this occurs the system is ready for the comparison with the input face from the visual system. It was also necessary to implement a process that loaded every image from known face database into a variable and substituted it as a function parameter. Next, function returned 128-number array for each image that represents the values of the individual points of the face in the image. These measurements are generated passing through the pre-trained NN.

\subsection{Face Detection}

Process is the precondition for successful face recognition. The method, chosen for the face detection is HOG. As an input for face detection will be face of a visitor captured by the camera.

\subsection{Face Recognition}

After successful face detection from the static image, the next step for algorithm is to recognize face of visitor. The recognition is successful, when there is a match between face of visitor and known faces in the database. The match, based on 128 face point numbers extracted by faceRecognition's faceEncodings function, is computed via compareFaces function. This function returns list of boolean values for each known face image. If a known face database contains 30 photos, the generated list will contain 30 boolean values. If the face matches, the true value is assigned to particular image.
During the implementation on Raspberry $\mathrm{Pi}$, the algorithm was continuously tested, and authors were able to observe the real-time output on the monitors screen. Sample output of the face recognition is depicted in Fig. 1.

\subsection{Output to Mobile Device}

After successful or unsuccessful recognition, the result is provided to the administrator. It includes the photo of a visitor, having possible name already assigned. Note that in case the visitor is unknown, instead of name, the statement "unknown person" is displayed. Once administrator receives a notification, the decision on the access control can be made. Two forms of control are available:

- Open - visitor is permitted to enter.

- Abort - the access is forbidden.

\section{TESTING AND EVALUATION OF IMPLEMENTED SOLUTION}

Multiple measurements have been made to determine the boundaries of an algorithm designed to detect and recognize a person's face (see Tab. 1). The system was tested on four subjects. Each of these was photographed, thus the system could be provided with input images representing known database of faces. Overall, there are 28 photos in the database, 7 for each person. To increase recognition success, it was necessary to provide images where the person is at different distances, under different lighting conditions, and where the face position is at minor angle to the camera's position.

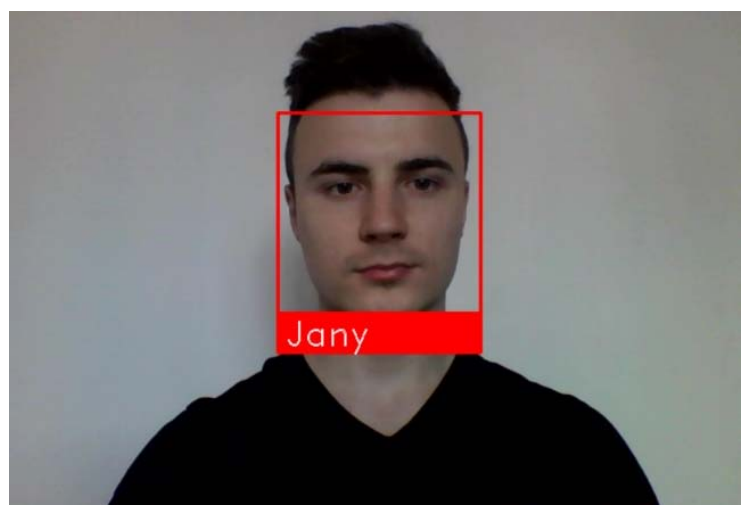

Fig. 1 Recognized face

Table 1 Detection and recognition success

\begin{tabular}{|c|c|c|c|}
\hline $\begin{array}{l}\text { Distance } \\
\text { of visitor }\end{array}$ & Face angle & $\begin{array}{c}\text { Successful } \\
\text { detection }\end{array}$ & $\begin{array}{l}\text { Successful } \\
\text { recognition }\end{array}$ \\
\hline $30 \mathrm{~cm}$ & direct & $100 \%$ & $100 \%$ \\
\hline $30 \mathrm{~cm}$ & 45 degrees & $83,33 \%$ & $100 \%$ \\
\hline $60 \mathrm{~cm}$ & direct & $100 \%$ & $100 \%$ \\
\hline $60 \mathrm{~cm}$ & 45 degrees & $83,33 \%$ & $100 \%$ \\
\hline $90 \mathrm{~cm}$ & direct & $100 \%$ & $100 \%$ \\
\hline $90 \mathrm{~cm}$ & 45 degrees & $83,33 \%$ & $91,66 \%$ \\
\hline $120 \mathrm{~cm}$ & direct & $100 \%$ & $91,66 \%$ \\
\hline $120 \mathrm{~cm}$ & 45 degrees & $75 \%$ & $83,33 \%$ \\
\hline
\end{tabular}




\subsection{Detection and Recognition Success Rate}

When a subject arrives at the door (visual input to the system), the position could appear at different distances, thus the following was tested (see Tab. 1):

1. The position of subject 30 centimeters away.

2. The position of subject 60 centimeters away.

3. The position of subject 90 centimeters away.

4. The position of subject 120 centimeters away.

For each distance and for each face angle, 3 images were created for each person. A different measurement angle is determined for each distance. During the image capturing, as the first, the subject had face facing camera directly, and then at angle of approx. 45 degrees.

Another parameter to be tested is the initialization time, that means the time, the software solution needs to calculate the values of the individual points on the faces and also to store the individual results in variables. The resulting time equals the initialization of all 28 photos from the database. The database contained photographs of different sizes and the time needed for initialization was estimated to 8 minutes and 20 seconds.

\subsection{Time required for Detection and Recognition}

This part is focused on the testing of the time per which the face has been detected and recognized. We should remember that face detection is a precondition for face recognition. The implemented system should also be able to detect multiple faces from the image. Therefore, the presence of several people was also tested. Detection and recognition time results are shown in Tab. 2.

Table 2 Average measured time for one subject at the time

\begin{tabular}{|c|c|c|c|}
\hline $\begin{array}{c}\text { Visitor's } \\
\text { distance }\end{array}$ & Face degree & Detection time & $\begin{array}{c}\text { Recognition } \\
\text { time }\end{array}$ \\
\hline $30 \mathrm{~cm}$ & direct & 9 seconds & 7 seconds \\
$30 \mathrm{~cm}$ & 45 degrees & 11 seconds & 7 seconds \\
\hline $60 \mathrm{~cm}$ & direct & 11 seconds & 8 seconds \\
$60 \mathrm{~cm}$ & 45 degrees & 12 seconds & 8 seconds \\
\hline $90 \mathrm{~cm}$ & direct & 11 seconds & 9 seconds \\
$90 \mathrm{~cm}$ & 45 degrees & 10 seconds & 8 seconds \\
\hline $120 \mathrm{~cm}$ & direct & 8 seconds & 8 seconds \\
$120 \mathrm{~cm}$ & 45 degrees & 11 seconds & 9 seconds \\
\hline
\end{tabular}

\subsection{Results of testing}

During testing of system functionality (Fig.2), speed and accuracy, it was found that the system can detect and recognize faces very accurately. The negative side of the results is the time that software solution takes to perform detection and recognition, whether for one person or two. The time needed for recognition has increased with the number of images in database. However, the number of faces in the database did not affect the time needed for face detection in any way. The time needed for face detection was not influenced by the angle of the face. Thus, the program handles detection at a relatively constant time. However, the success of detection varied significantly with the face angle change. The threshold was approximately 45 degrees. If the face was at even greater angle, the algorithm worked with a smaller part of the face, resulting in a problem with detection. Some photographs were taken with less illumination; this did not affect the result nor time of detection and recognition.

Based on the test results, we can conclude that face detection and recognition operate with high precision. The system took into account that the face of subject may not always be directly opposite the camera but may have some angle. At the 45 -degree angle, the success rate was $81.22 \%$. If a subject was looking directly at the camera, the face was detected with a $100 \%$ success rate. Face recognition in case of the direct face position achieved $97.92 \%$. The success rate at 45 -degrees angle was $93.74 \%$.

If we calculate the time of detection and recognition, we realize that the person in front of the input device has to wait almost 20 seconds before the result. When two faces are detected simultaneously, the detection time has increased by approximately 2 seconds. In subsequent recognition, time increased by less than a second. The difference between the detection time of one and two faces is relatively neglected. The system initialization time was 8 minutes and 20 seconds. During the real use, such time does not have a negative impact on the quality of the device, as the system is only initialized after the initial start-up.

All results have been summarized. Success rate results have been achieved as follows, average face:

- detection: subject looking directly to the camera, success rate $100 \%$,

- detection: angle 45 degrees, success rate $81,22 \%$,

- overall detection success rate is $90,61 \%$,

- recognition: subject looking directly to the camera, success rate $97,92 \%$,

- recognition: angle 45 degrees, success rate $93,74 \%$,

- overall recognition success rate is $95,83 \%$.

Results of detection and recognition time were achieved as follows:

- $\quad$ average face detection time: 10,38 seconds,

- average face detection time of two faces: 12,25 seconds,

- $\quad$ average face recognition time: 7,13 seconds,

- average face recognition time of two faces: 8 seconds,

- recognition time of two faces: 8 seconds.

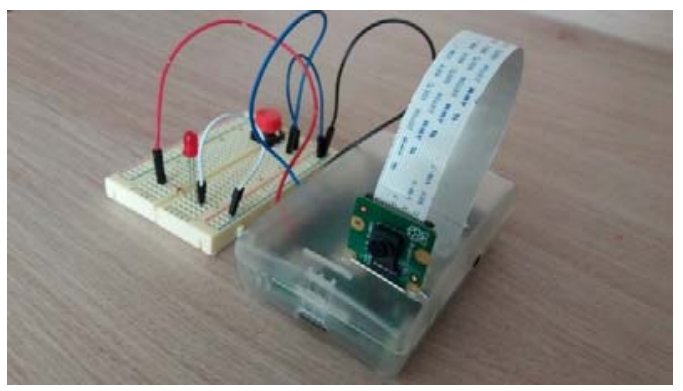

Fig. 2 Experimental hardware solution

\section{CONCLUSIONS}

The main goal of the research was a hardware-software implementation of experimental Raspberry-based access control system. This solution contains a camera that allows the system to detect and recognize the face of person. Face 
detection starts automatically when visitor presses the button. The administrator is then notified on the recognition result.

Based on the proposal, experimental implementation of the such system was carried out. The system consists of several parts. The first part includes initialization of images, i.e. preparing images for the database of known faces, via extraction of facial points, using a pre-trained neural network. Another part of the system is face detection and recognition. The face of person captured by Raspberry camera is compared to each known face for recognition after successful detection. Subsequently, the administrator is notified and can provide commands to electronic door lock system.

System testing proved a high detection rate of $90.61 \%$ and facial recognition rate of $95.83 \%$. Positive results have been achieved at different distances from the camera, at different angles of face, lightning conditions, and multiple test subjects at the time.

System presented in this paper is part of the overall solution for the access control, in the next steps the proposed solution is to be connected with the real doorlocking device, proving semiautomated approach to control of entry to specific premises.

\section{ACKNOWLEDGMENTS}

This publication is the result of the Project implementation: University Science Park TECHNICOM for Innovation Applications Supported by Knowledge Technology, Phase II., ITMS: 313011D232, supported by the Research \& Inovation Operational Programme funded by the ERDF. We support research activities in Slovakia/This project is being co-financed by the European Union.

\section{REFERENCES}

[1] PAPCUN, P. - KAJÁTI, E. - ZOLOTOVÁ, I.: "IoT and cloud technology as ubiquitous computing in case study of intelligent household" International Journal of Computers, 2017.

[2] HAFIZ, A. - ISHRAQ, A. - MANIRUL, I.: "HOGCNN Based Real Time Face Recognition." International Conference on Advancement in Electrical and Electronic Engineering (ICAEEE), 2018.

[3] ISLAM, T. - RAJ, G. R. - AL-MURAD, A.: "Performance of SVM, CNN, and ANN with BoW, HOG, and Image Pixels in Face Recognition." International Conference on Electrical \& Electronic Engineering, 2017.

[4] VIOLA, P. - JONES, M.: "Rapid Object Detection Using a Boosted Cascade of Simple Features." Proceedings of IEEE Computer Society Conference on Computer Vision and Pattern Recognition. CVPR 2001.

[5] CUIMEI, L. - ZHILIANG, Q. - NAN, J. - JIANHUA, W.: "Human Face Detection Algorithm via Haar Cascade Classifier Combined with Three Additional
Classifiers." International Conference on Electronic Measurement \& Instruments (ICEMI), 2017.

[6] VARSHNEY, P. K. - ARORA, M. K.: "Advanced Image Processing Techniques for Remotely Sensed Hyperspectral Data." Berlin: Springer-Verlag, 323 p, 2004.

[7] HUANG, L.: 2007. "Variable selection in multi-class support vector machine and applications in genomic data analysis." Ann Arbor: ProQuest Information and Learning Company. 129 p. Dissertation/Thesis, 2008.

[8] KOTSIA, I. - PITAS, I.: "Real time facial expression recognition from image sequences using support vector machines". IEEE International Conference on Image Processing, (ICIP), 2005.

[9] SHU, S. - LUO, J. - SUN, B. - YU, N.: “Color Images Face Recognition Based on Multi-classification Support Vector Machine". International Conference on Computer Science and Education, 2010.

[10] LAL, M. - KUMAR, K. - ARAIN, R. H. - MAITLO, A. - RUK, S. A. - SHAIKH, H.: "Study of Face Recognition Techniques: A Survey." International Journal of Advanced Computer Science and Applications, 2018.

[11]NAJARIAN, K. - SPLINTER, R.: "Biomedical Signal and Image Processing: Second Edition”. Boca Raton: CRC Press. 411 p., 2012.

Received May 5, 2019, accepted September 24, 2019

\section{BIOGRAPHIES}

Ondrej Kainz was born in 1988. In 2017 received PhD degree in Computer Science from the Technical university of Kosice. Since the very same year he is the assistant professor at the Department of Computers and Informatics of the Faculty of Electrical Engineering and Informatics (FEEI) at the Technical university of Kosice. His scientific research interests include computer vision, image processing and computer networks.

Ján Drozd is a master's degree student of the Informatics at the Technical University of Kosice. In 2017 he graduated (Bc) the same study program at the department of Computers and Informatics of the FEEI at Technical University in Košice.

Miroslav Michalko received his M.Sc. and Ph.D. degree in Informatics from Technical University in Kosice (Slovakia). For more than 10 years he is member of well recognized research institution - Computer Networks Laboratory at Department of Computers and Informatics at Technical University of Kosice (DCI TUKE). Now he is an Assistant Professor at DCI TUKE and his lectures are focused on Computer Networks, he is more than 10 years active instructor in Cisco Networking Academy. His research includes multimedia content delivery, video streaming services, web and cloud services, multimedia on mobile devices, innovative teaching\&learning techniques and IoE/IoT solutions. 
František Jakab was born in 1959. Graduated from St. Petersburg Electro technical University, PhD degree received from Technical university of Kosice \& is associated professor since 2008. He has extensive experience in networking and utilization of ICT in education where he established well known research centre - Computer Networks Laboratory. He has been a coordinator of several large international projects, coordinator of national wide ICT projects and research grants. Involved into Cisco Networking academy program in Slovakia and since 2001 in position of coordinator of Program in Slovakia, 2008-2014 He was awarded as a "IT person of the year 2006" in Slovakia. 